\title{
Influence comparée de la saison sur la phénologie et sur les dégâts d'insectes phyllophages chez de jeunes individus d'arbres de forêt tropicale en Guyane française
}

\author{
Pascal Gombauld, Judy Rankin-de Mérona
}

Station de recherches forestières, Inra BP 709, 97387 Kourou cedex, Guyane française

(Reçu le 29 juillet 1996 ; révisé le 15 novembre 1996 ; accepté le 21 août 1997)

\begin{abstract}
Influence of season on phenology and insect herbivory on saplings of tropical rain forest trees in French Guiana. Leaf herbivory by insects as a function of season was quantified for saplings of five major tree species of a tropical rain forest in French Guiana. We determined the impact of herbivory based on the observed ratio of leaf area eaten to total leaf area (attack ratio) in a study of individual leaves on young saplings naturally established in undisturbed and adjacent logged forest. Measurements were conducted during the strongly contrasted dry and rainy seasons over a period of 10 months. The five species chosen for the study are Dicorynia guianensis, Eperua falcata, Eperua grandiflora, Goupia glabra and Qualea rosea. For four of the five species, leaf production varies significantly with season. D. guianensis is the only species of the five for which leaf production is maintained throughout the dry season. This has the effect of diminishing the relative impact of herbivory during a period when ground water deficits create conditions unfavorable for plant growth. Three other species experiencing high levels of herbivory during the dry season have significantly lower mean height growth than during the rainy season. In contrast, D. guianensis and $G$. glabra have similar height growths regardless of season. Seasonal variation in leaf production and leaf attack also occur in similar proportions. The resulting attack ratios, with the exception of $E$. grandiflora, are not significantly different between the rainy season and dry season observations. This supports the hypothesis that, for the tropical rain forest, the rainy season is the more favorable period for leaf-eating insect activity. The influence of leaf production phenology on the population dynamics of leaf-eating insects in this community is also discussed. (@ Inra/Elsevier, Paris.)
\end{abstract}

herbivory / insect / phenology / seasonality / tropical rain forest

Résumé - Des observations conduites en forêt tropicale humide, dans la zone littorale de la Guyane Française, ont permis de quantifier, en fonction des saisons, les attaques par les insectes phyllophages et la phénologie de jeunes individus de cinq espèces d'arbres (Dicorynia guianensis, Eperua falcata, Eperua grandiflora, Goupia glabra, Qualea rosea). D guianensis est la seule

E-mail : gombauld.p@cirad.fr ; merona.j@cirad.fr 
des cinq espèces étudiées chez laquelle la production foliaire est maintenue en saison sèche, ce qui tend à diminuer l'impact des phyllophages durant une période où l'alimentation hydrique des plantes est généralement peu favorable à la croissance. Les espèces ayant de fortes proportions de feuilles attaquées en saison sèche ont durant cette saison une croissance en hauteur significativement plus faible qu'en saison des pluies ( $E$. falcata, G. glabra et $Q$. rosea). Au contraire $D$. guianensis et $G$. glabra présentent des croissances en hauteur semblables en saison des pluies et en saison sèche. Les variations saisonnières de production foliaire et d'attaques d'insectes phyllophages s'effectuent dans des proportions semblables. Le taux de défoliation qui en résulte (sauf pour E. grandiflora) n'est pas significativement différent entre la saison sèche et la saison des pluies. Notre étude conforte l'hypothèse selon laquelle la saison des pluies serait favorable à l'activité des insectes phyllophages en forêt tropicale humide. L'impact de la phénologie de la production foliaire sur la dynamique des populations de phyllophages est également discuté. (C Inra/Elsevier, Paris.)

\section{phyllophagie / insecte / phénologie / saisonnalité / forêt tropicale humide}

\section{INTRODUCTION}

Les communautés végétales et leurs cohortes d'herbivores sont les unes et les autres soumises aux fluctuations des facteurs abiotiques (parmi eux, la lumière, les éléments minéraux, l'eau, la température, le vent) $[3,5,7,18,23]$. Étudier l'influence d'un de ces facteurs, simultanément sur la phénologie des plantes et les attaques d'insectes phyllophages est une manière efficace de prendre en compte les interactions entre les composantes animale et végétale $[4,15,16,24$, 31, 32]. De ce point de vue, en forêt tropicale humide, les études concernant la lumière sont nombreuses $[9,10,17,30$, $34,39]$, tandis que la saison n'est pas souvent abordée [16, 35]. En milieu tropical la température et la durée de la journée solaire étant plus ou moins constantes au cours de l'année, les différences saisonnières se manifestent essentiellement à travers la pluviométrie [11]. Les études relatives à l'influence de la saison sur les populations d'herbivores sont peu nombreuses et il n'existe pas pour les forêts tropicales humides de modèles permettant de prédire leur dynamique ; cependant il est admis par la plupart des biologistes travaillant en milieu tropical, que les populations d'insectes présentent d'im- portantes variations saisonnières [12] Les résultats concernant l'influence de la pluviométrie sur la phénologie des espèces végétales forestières sont plus nombreux mais parfois contradictoires [ 8 , 21, 22, 36]. Ainsi, au Costa Rica, dans la station de recherche La Selva en forêt tropicale, où les saisons sont peu marquées, mais différentiables comme en Guyane Française, la production foliaire de Piper arieianum est indépendante des précipitations [27]. Au contraire, Aide [1], sous des latitudes voisines, met en évidence sur l'île de Barro Colorado à Panama, un phénomène d'échappement aux phyllophages par augmentation de la production foliaire pendant la saison sèche pour Hybanthus prunifolius.

Ainsi, à ce jour, les interférences entre les facteurs environnementaux, l'intensité des dégâts de phyllophages et la phénologie restent-elles encore à explorer pour les arbres de forêt tropicale humide. Dans le but de clarifier et de tester l'hypothèse selon laquelle la saison influence, en forêt tropicale, les relations trophiques entre les insectes phyllophages et leurs plantes nourricières, nous avons conduit à Paracou, en Guyane Française, une étude comparée de la phénologie de jeunes plants de cinq essences forestières et de leur attaque par ces insectes. 


\section{MATÉRIEL ET MÉTHODES}

Le dispositif expérimental de Paracou est situé sur la zone littorale de la Guyane $\left(5^{\circ} 15^{\prime} \mathrm{N}, 52^{\circ} 55^{\prime} \mathrm{O}\right)$ et s'étend sur un site de 500 ha. Sur cette station de recherche sont localisées des parcelles ( 9 ha) de forêt naturelle, dont certaines ont subi différentes intensités de traitement sylvicole $[6,33]$. Au sein de ce site, deux parcelles furent retenues car la régénération naturelle des espèces étudiées y était suffisante pour la réalisation de ce travail. L'étude a porté sur cinq essences forestières, d'intérêt commercial majeur, présentant des comportements variés vis-à-vis de la lumière : Dicorynia guianensis Amshoff (Caesalpiniaceae) ou Angélique, Eperua falcata Aublet (Caesalpiniaceae) ou Wapa, Eperua grandiflora (Aublet) Bentham (Caesalpiniaceae) ou Wapa, Goupia glabra Aublet (Goupiaceae) ou Goupi et Qualea rosea Aublet (Vochysiaceae) ou Gonfolo. À l'âge adulte, ces cinq espèces appartiennent à l'étage dominant de la forêt naturelle. Dicorynia guianensis, Eperua falcata et Eperua grandiflora sont des espèces semi-tolérantes au couvert forestier [14]. Qualea rosea est une espèce opportuniste favorisée par les traitements sylvicoles forts [29]. Goupia glabra est une espèce pionnière dont le recrutement se fait en condition de forte lumière exclusivement ; les semis de Goupia glabra ne sont pas tolérants au sousbois forestier.

L'échantillon était composé au départ de 340 individus (68 par espèce) issus de la régénération naturelle, et déjà établis. Ces plants, pris au hasard parmi ceux de conformation normale et de taille inférieure à $2 \mathrm{~m}$, furent étiquetés et chacune de leurs feuilles numérotée (pour D. guianensis, E. falcata et E. grandiflora qui possèdent des feuilles composées, chaque foliole fut numérotée). La hauteur fut mesurée pour chacun des plants et l'intensité des dégâts d'insectes pour chacune des feuilles. La hauteur fut mesurée en $\mathrm{cm}$, du sol au bourgeon terminal. Pour chaque feuille, la surface consommée par les insectes et la surface totale furent mesurées à l'aide d'une grille (unité élémentaire de mesure : $0,25 \mathrm{~cm}^{2}$ ) appliquée contre la feuille. Il sagit de la méthode la plus précise utilisée jusquà présent pour estimer l'une et l'autre sans destruction des feuilles $[2,9,26]$. Les différentes tech- niques de mesure des dégâts de phyllophages sont présentées et discutées par Lowman [25].

Les mesures se sont déroulées en trois campagnes (mai-juin 1993, août-septembre 1993 et janvier-février 1994). Les données météorologiques (figurel) issues de la station Paracou, à $6 \mathrm{~km}$ des parcelles, indiquent que de mai à juillet la pluviométrie fut en moyenne de $297,8 \mathrm{~mm} / \mathrm{mois}$, et d'août à décembre de $137,5 \mathrm{~mm} / \mathrm{mois}$. Ces deux périodes correspondent respectivement à la grande saison des pluies et à la grande saison sèche. La hauteur et les surfaces attaquées furent mesurées une fois par campagne pour chacun des plants échantillonnés. À partir des trois relevés furent établies les variables suivantes pour chacune des saisons considérées: nombre de feuilles produites, nombre de feuilles tombées, croissance en hauteur (en $\mathrm{cm}$ ), proportion de feuilles attaquées et taux de défoliation (rapport entre la surface enlevée par les insectes et la surface foliaire du plant supposé indemne d'attaque).

Les tests non-paramétriques de KruskallWallis et de Wilcoxon furent utilisés respectivement pour les comparaisons multiples, et les comparaisons de séries appariées (variables dépendantes). Le test de Kolmogorov-Smirnov fut utilisé pour des séries supposées indépendantes. Les pourcentages furent comparés à l'aide d'un test du chi-carré $\left(\chi^{2}\right)$.

\section{RÉSULTATS}

\subsection{Phénologie des plants}

\subsubsection{Production foliaire}

La production foliaire, estimée en nombre de feuilles, est significativement différente entre les espèces étudiées, aussi bien en saison des pluies qu'en saison sèche (Kruskall-Wallis : $p<0,001$ ). G. glabra possède une production foliaire plus forte que les quatre autres espèces en saison des pluies et en saison sèche (tableau I). En saison des pluies, $Q$. rosea possède une production foliaire plus éle- 


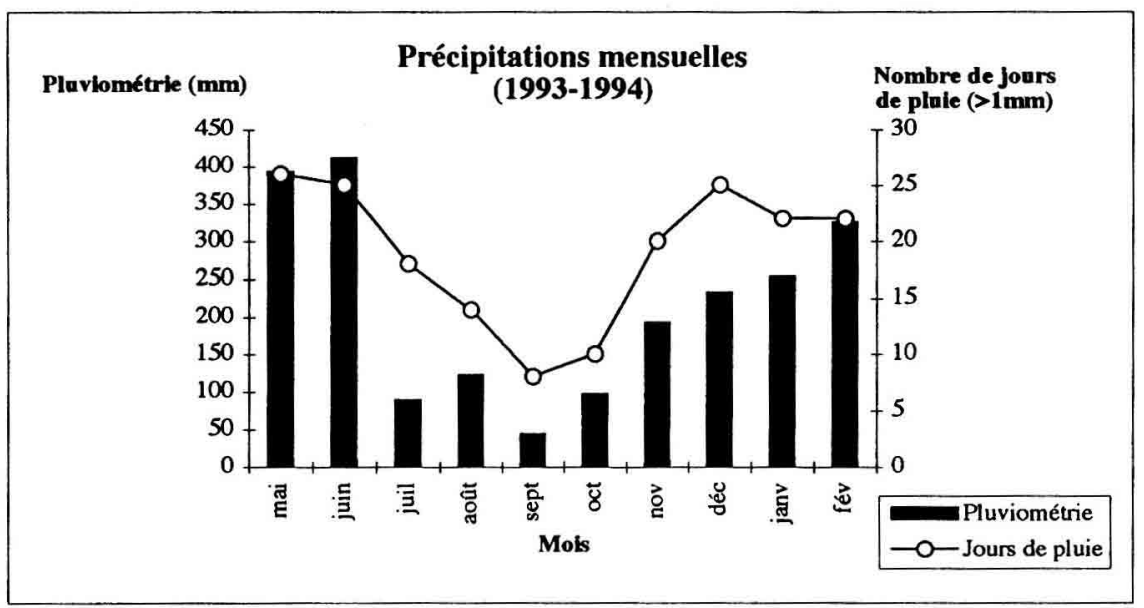

Figure 1. Pluviométrie.

vée que $D$. guianensis, E. falcata et $E$. grandiflora.

Chez quatre espèces, la production foliaire est significativement plus forte en saison des pluies (Wilcoxon : E. falcata et $Q$. rosea $p<0,001 ; E$. grandiflora et $G$. glabra $p=0,05)$. $D$ guianensis suit la même tendance, mais la différence nest pas significative entre saison des pluies et saison sèche (Wilcoxon : $p=0,892$ ).

\subsubsection{Chute des feuilles}

La chute des feuilles est significativement différente entre les espèces aussi bien en saison des pluies qu'en saison sèche (Kruskall-Wallis : $p<0,001$ ). $G$. glabra présente la chute foliaire moyenne la plus forte en saison des pluies et en saison sèche. $Q$. rosea présente une chute foliaire moyenne plus forte que celles de $D$. guianensis, E. falcata et E. grandiflora en saison des pluies et en saison sèche (tableau $I$ ).

G. glabra présente une chute foliaire moyenne significativement plus impor- tante en saison des pluies (Wilcoxon: $p=0,003$ ). Pour quatre espèces, la chute foliaire moyenne ne diffère pas significativement entre saison des pluies et saison sèche (Wilcoxon : $D$. guianensis $p=0,454 ;$ E. falcata $p=0,134 ;$ E. grandiflora $p=0,815 ; Q$. rosea $p=0,386$ ).

\subsubsection{Croissance en hauteur}

En saison des pluies et saison sèche, la croissance en hauteur est significativement différente entre les espèces (Kruskall-Wallis : $p<0,001$ ). En saison des pluies, $E$. grandiflora et $G$. glabra possèdent les croissances en hauteur les plus fortes. En saison sèche, G. glabra possède la croissance la plus forte (tableau I).

Chez deux espèces, la croissance en hauteur ne diffère pas significativement entre saison des pluies et saison sèche (Wilcoxon : D. guianensis $p=0,672 ; G$. glabra $p=0,777)$. E. falcata, $E$ grandiflora et $Q$. rosea présentent une croissance en hauteur significativement plus 
élevée en saison des pluies (Wilcoxon : E. falcata $p<0,001 ; E$. grandiflora $p=0,004 ; Q$. rosea $p=0,035$ ).

\subsection{Dégâts dinsectes phyllophages}

\subsubsection{Proportions de feuilles attaquées}

Toutes espèces confondues, le pourcentage de feuilles attaquées varie significativement en fonction des saisons $\left(\chi^{2}=48,35 p<0,001\right)$. Chez D. guianensis et $G$. glabra, ce pourcentage ne varie pas significativement en fonction des saisons (D. guianensis $\chi^{2}=0,53 p=0,465$; G. glabra $\left.\chi^{2}=0,52 p=0,469\right)$, alors que chez $E$. falcata, E. grandiflora et $Q$. rosea, il est significativement plus élevé en saison sèche ( $E$. falcata $\chi^{2}=12,83$ $p<0,001 ;$ E. grandiflora $\chi^{2}=34,97$ $p<0,001 ; Q$. rosea $\left.\chi^{2}=5,07 p=0,024\right)$ (tableau II).

\subsubsection{Taux de défoliation}

Le taux de défoliation est significativement différent entre les espèces tant en saison des pluies qu'en saison sèche (Kruskall-Wallis : $p<0,001$ ). En saison des pluies et en saison sèche, G. glabra présente le taux de défoliation le plus faible des cinq espèces. Les plus forts taux de défoliation sont supportés par $D$. guianensis en saison des pluies, et par $D$. guianensis et E. grandiflora en saison sèche (tableau III). Le rapport entre les taux de défoliation extrêmes est de l'ordre de 4 en saison des pluies et de cing en saison sèche.

Seul E. grandiflora présente un taux de défoliation significativement différent entre saison des pluies et saison sèche (Kolmogorov-Smirnov : $p<0,001$ ). Chez D. guianensis, E. falcata, G. glabra et $Q$. rosea, le taux de défoliation n'est pas significativement différent entre les saisons (Kolmogorov-Smirnov : D. guianensis $p=0,559 ;$ E. falcata $p=0,065 ; G$. glabra $p=0,699 ; Q$. rosea $p=0,149)$ (tableau III).

\section{DISCUSSION}

La production foliaire est plus élevée chez quatre espèces ( $E$. falcata, $E$. grandiflora, $G$. glabra et $Q$. rosea) sur cinq en saison des pluies. La chute foliaire n'est pas influencée par la saison, sauf chez $G$. glabra, qui présente une chute foliaire plus élevée en saison des pluies. La croissance en hauteur est plus forte en saison des pluies chez trois des cinq espèces $(E$. falcata, $E$. grandiflora et $Q$. rosea).

Chez D. guianensis, ni la croissance ni la production foliaire ne sont, dans nos conditions d'étude, influencées par la saison. La proportion de feuilles attaquées et le taux de défoliation ne varient pas non plus en fonction de la saison. Chez $D$. guianensis, contrairement aux quatre autres espèces, la production foliaire se maintient à un niveau aussi élevé en saison sèche qu'en saison des pluies. Ce comportement confirmerait l'hypothèse de Aide [1] selon laquelle certaines espèces augmentent ou maintiennent leur production foliaire durant la saison sèche, ce qui a pour effet de limiter l'impact des phyllophages.

Chez E. falcata, la croissance, la production foliaire et la consommation foliaire par les insectes sont plus importantes en saison des pluies. La production foliaire et les dégâts d'insectes sont environ quatre fois plus élevés en saison des pluies qu'en saison sèche (tableau $I V$ ). En conséquence, le taux de défoliation ne varie pas de façon significative entre les saisons (tableau III). Nous suggérons que cette espèce trouve en saison des pluies des conditions favorables à sa croissance lui permettant une accumulation de biomasse malgré la forte activité des insectes durant cette période. Au contraire, certainement du fait de la faible production 


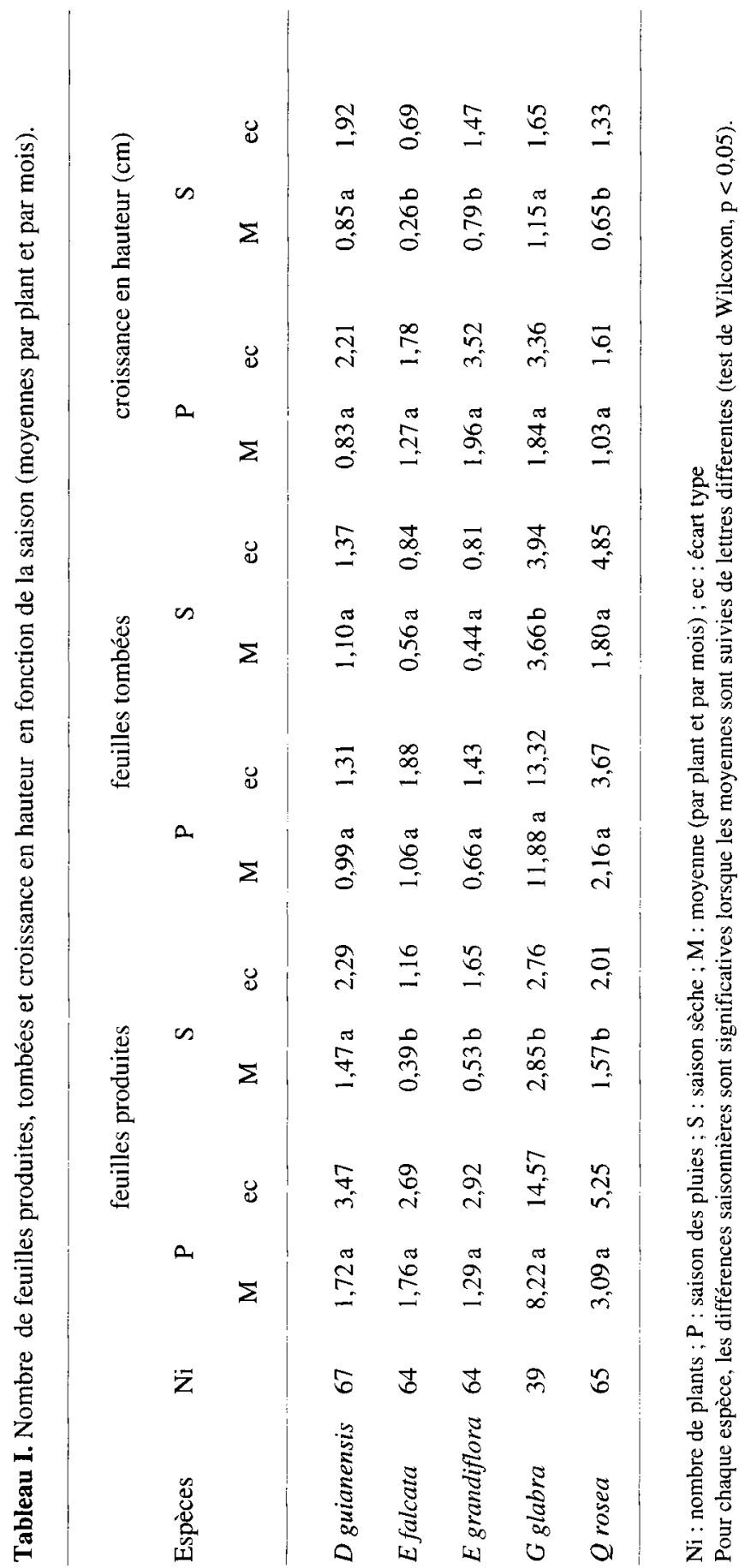


Tableau II. Proportion de feuilles attaquées en fonction de la saison

\begin{tabular}{|c|c|c|c|c|c|c|c|c|c|}
\hline \multirow[b]{2}{*}{ Espèces } & \multirow[b]{2}{*}{$\mathrm{Ni}$} & \multicolumn{4}{|c|}{ Saison des pluies } & \multicolumn{4}{|c|}{ Saison sèche } \\
\hline & & $\mathrm{Nf}$ & $\mathrm{Na}$ & $\%$ & & $\mathrm{Nf}$ & $\mathrm{Na}$ & $\%$ & \\
\hline$D$ guianensis & 67 & 345 & 254 & 74 & a & 491 & 349 & 71 & a \\
\hline$E$ falcata & 64 & 338 & 139 & 41 & a & 126 & 76 & 60 & b \\
\hline E grandiflora & 64 & 247 & 96 & 39 & a & 170 & 117 & 69 & b \\
\hline$G$ glabra & 39 & 962 & 417 & 43 & $\mathbf{a}$ & 555 & 252 & 45 & a \\
\hline$Q$ rosea & 65 & 603 & 409 & 68 & a & 511 & 379 & 74 & b \\
\hline
\end{tabular}

$\mathrm{P}$ : saison des pluies; $\mathrm{S}$ : saison sèche; $\mathrm{Ni}$ : nombre de plants; $\mathrm{Nf}$ : nombre de feuilles produites; $\%:(\mathrm{Na} / \mathrm{Nf})^{*} 100 ; \mathrm{Na}:$ nombre de feuilles attaquées parmis $\mathrm{Nf}$

foliaire, la proportion de feuilles attaquées est significativement plus élevée en saison sèche, augmentant ainsi l'impact relatif de la phyllophagie; en effet en saison sèche, la croissance en hauteur moyenne des plants d'E. falcata est significativement plus faible qu'en saison des pluies. Chez $Q$. rosea, les phénomènes sont sensiblement les mêmes que chez $E$. falcata.

Chez E. grandiflora, la saison des pluies favorise la production foliaire et la croissance en hauteur des plants, mais n'a pas d'influence sur la chute des feuilles. La consommation foliaire par les insectes semble beaucoup plus élevée en saison sèche (tableau III). Cependant, l'essentiel des attaques sont dues à des Psyllidae (Homoptera) présents surtout en saison des pluies; à cette époque de l'année leurs attaques consistant en des prélèvements de sève par piqûre, ne provoquent pas de diminutions de la surface foliaire. Celles-ci n'apparaissent que plus tard,

Tableau III. Taux de défoliation par individu et par espèce en fonction de la saison

\begin{tabular}{|c|c|c|c|c|c|}
\hline \multirow[b]{2}{*}{ Espèces } & \multirow[b]{2}{*}{$\mathrm{Ni}$} & \multicolumn{2}{|c|}{ Saison des pluies } & \multicolumn{2}{|c|}{ Saison sèche } \\
\hline & & M & ec & M & ec \\
\hline$D$ guianensis & 67 & $19,69 \mathrm{a}$ & 25,84 & $20,19 \mathrm{a}$ & 25,50 \\
\hline E falcata & 64 & 9,41 a & 18,22 & $10,75 \mathrm{a}$ & 18,18 \\
\hline E grandiflora & 64 & 9,32 a & 19,71 & $23,89 b$ & 27,02 \\
\hline$G$ glabra & 39 & $5,00 \quad a$ & 10,99 & 4,87 a & 10,43 \\
\hline$Q$ rosea & 65 & $11,41 \mathrm{a}$ & 21,73 & $11,63 \mathrm{a}$ & 17,80 \\
\hline
\end{tabular}

$\mathrm{Ni}$ : nombre de plants; $\mathrm{M}$ : taux de défoliation moyen ; ec : écart type 
Tableau IV. Proportion entre saisons (P/S) des surfaces foliaires produites et attaquées

\begin{tabular}{llll}
\hline Espèces & $\mathrm{Ni}$ & SFOn (P/S) & SFOa (P/S) \\
D guianensis & 67 & 1,21 & 1,14 \\
E falcata & 64 & 4,35 & 3,91 \\
E grandiflora & 64 & 2,17 & 0,95 \\
G glabra & 39 & 2,74 & 2,97 \\
Q rosea & 65 & 1,95 & 1,93 \\
\hline
\end{tabular}

$\mathrm{Ni}$ : nombre de plants ; SFOn (P/S) : Rapport entre la surface foliaire produite durant la saison des pluies et la surface foliaire produite durant la saison sèche ; $\mathrm{SFOa}(\mathrm{P} / \mathrm{S})$ : Rapport entre la surface foliaire attaquée durant la saison des pluies et la surface foliaire attaquée durant la saison sèche.

après la maturation foliaire, sous forme de nécroses concentriques bien caractéristiques. Ainsi, les fortes attaques mesurées en saison sèche ont-elles en fait été effectuées pour partie en saison des pluies. Le taux de défoliation significativement plus faible en saison des pluies qu'en saison sèche résulte d'une forte production foliaire combinée à de faibles diminutions des surfaces foliaires au cours de cette saison (même si les attaques sont en réalité effectuées durant celle-ci). La diminution de surface foliaire s'opérant réellement en saison sèche, l'impact des phyllophages se trouve donc, comme chez $E$. falcata, accentué durant cette saison. Comme chez $E$. falcata également, forte production foliaire et fortes attaques d'insectes (Homoptères Psyllidae) se produisent simultanément en saison des pluies.

Chez $G$. glabra, la production et la chute foliaires sont plus importantes en saison des pluies qu'en saison sèche. Le renouvellement des feuilles est donc plus élevé en saison des pluies. À condition que les feuilles produites ne soient pas plus sensibles à cette période, ce fort renouvellement des feuilles constitue pour la plante un mode de défense indirecte, car il y a augmentation de la surface photosynthétique intacte. La croissance en hauteur comme la proportion de feuilles attaquées sont indépendantes de la saison. Les surfaces produites et attaquées sont environs trois fois plus importantes en saison des pluies (tableau $I V$ ) et, comme chez E. falcata, le taux de défoliation n'est pas significativement différent entre saison des pluies et saison sèche.

La production foliaire et les attaques d'insectes s'observent principalement en saison des pluies, excepté chez $D$. guianensis et $G$. glabra, sur lesquels l'activité des insectes est sensiblement la même quelle que soit la saison (tableau IV). Les taux de défoliation ne diffèrent pas de façon significative entre les saisons (sauf chez E. grandiflora) car, lorsque la production foliaire varie, les surfaces prélevées par les insectes varient dans le 
même sens et dans des proportions voisines. Le taux de défoliation n'est donc pas un bon indicateur de l'influence de la saison sur la phénologie des plantes et sur l'activité consommatrice des phyllophages.

L'influence de la saison sur la proportion de feuilles attaquées varie. Nos résultats mettent en évidence un lien entre proportion de feuilles attaquées et croissance en hauteur. En effet, lorsque la proportion de feuilles attaquées est élevée, c'est-àdire en saison sèche dans notre étude, la croissance en hauteur est significativement plus faible (D. guianensis et $G$. glabra, tableaux I et $I I)$. À l'inverse, ni la proportion de feuilles ni la croissance en hauteur ne diffèrent significativement entre saisons chez les trois autres espèces étudiées (E. falcata, E. grandiflora et $Q$. rosea, tableaux $I$ et $I I)$. Notons que ces résultats sont relatifs aux conditions climatiques propres à la période d'étude. En 1993, la différence entre saison sèche et saison des pluies a été peu marquée du fait de la reprise des précipitations dès le mois de novembre. Il est concevable que les phénomènes décrits puissent être modifiés par un régime pluviométrique différent. En effet, des conditions de stress hydrique (saison sèche marquée, mauvais enracinement des plantules, faible rétention en eau du sol, forte éclaircie...) pourraient limiter la faculté des jeunes plantes à augmenter leur production foliaire et donc à compenser l'impact des diminutions de surface photosynthétique occasionnées par les attaques d'insectes phyllophages [13]. Nous émettons l'hypothèse que, dans nos conditions d'étude, ces attaques contribuent aux retards de croissance en hauteur observés chez les plantules d'E. falcata, $G$. glabra et $Q$. rosea en saison sèche. Ainsi, même si la pression des insectes phyllophages est plus importante en saison des pluies, l'impact des attaques serait plus élevé en saison sèche. Cette hypothèse n'a pas jusqu'à présent été démontrée.
Cependant, indépendamment de la saison, il existerait un lien entre taux de croissance et dégâts d'insectes phyllophages [8].

En conclusion, ce travail met en évidence une forte liaison entre un facteur abiotique, la pluviométrie, et la phénologie de quatre espèces d'arbres, mais aussi entre ce même facteur abiotique et l'activité des insectes phyllophages. Nous avons vu, à travers cette étude, qu'il existe des variations saisonnières de la production foliaire et des attaques d'insectes phyllophages. Ces variations s'effectuent dans des proportions semblables. Le taux de défoliation qui en résulte (sauf pour E. grandiflora) n'est donc pas significativement différent entre les saisons. La saison des pluies considérée comme limitante pour les insectes s'alimentant à la surface des feuilles [28] ne le serait pas pour les phyllophages majeurs de quatre des cinq espèces étudiées. En effet, nos résultats confirment l'abondance de certains arthropodes en saison des pluies $[20,37,38]$. Sans parler de tendance générale, notre étude révèle une action globalement favorable de la saison des pluies sur la production foliaire et la croissance, qui atténuent ainsi l'impact des attaques par les insectes. On peut donc s'attendre, pour bon nombre d'espèces, à un effet négligeable de ces derniers (dans le cas de plants déjà installés) en saison des pluies et à un effet plus ou moins sévère en fonction du stress hydrique subi par les plantules, en saison sèche. Nous avons montré que la phyllophagie se manifeste essentiellement lorsque la nourriture est disponible en grande quantité, c'est-à-dire pendant la saison des pluies, période de forte production foliaire. Nous émettons l'hypothèse que la saison n'agit pas seulement directement sur les populations de phyllophages en région tropicale humide, mais aussi et surtout indirectement à travers les variations saisonnières de production foliaire des plantes nourricières. Bien 
qu'il soit difficile d'établir une relation de cause à effet entre phénologie végétale et dégâts de phyllophages, étant données les coévolutions existant entre communautés animales et végétales $[19,31]$, nous pouvons, au vu de nos résultats, émettre l'hypothèse que la phénologie de la production foliaire est un facteur de contrôle de la dynamique des populations d'insectes phyllophages en forêt tropicale humide.

\section{REMERCIEMENTS}

Nous tenons à remercier vivement $\mathrm{P}$ Montpied qui a initié ce sujet de recherche. Sont également remerciés les chercheurs de la station de recherches forestières de l'Inra-Kourou, à savoir $\mathrm{T}$. Barigah, M. Béreau et $\mathrm{H}$. Caron pour les précieux conseils fournis, ainsi que $F$. Caillez, P. Cassier, A. Roques et H. Vasconcelos, pour la relecture de ce texte. Une attention toute particulière est adressée à l'équipe technique composée de P. Audin, D. Duchant, S. Dufort et P. Imbert qui ont participé aux campagnes de mesures.

\section{RÉFÉRENCES}

[1] Aide T.M., Dry season leaf production : an escape from herbivory, Biotropica 24 (4) (1992) 532-537.

[2] Aide T.M., Patterns of leaf development and herbivory in a tropical understory community, Ecology 74 (2) (1993) 455-466.

[3] Alexandre D.Y., Comportement hydrique au cours de la saison sèche et place dans la succession de trois arbres guyanais : Trema micrantha, Goupia glabra et Eperua grandiflora, Ann. Sci. For. 48 (1991) 101-112.

[4] Anderson C., Lee S.Y., Defoliation of the mangrove Avicennia marina in Hong Kong: cause and consequences, Biotropica 27 (2) (1995) 218-226.

[5] Barigah T.S., Huc R., Imbert P., Assimilation nette foliaire et croissance de quelques espèces de la forêt tropicale humide guyanaise cultivées sous différents régimes lumineux, Ann. Sci. For. 55 (6) (1998) 681-706.
[6] Bariteau M., La régénération naturelle avant et après exploitation sur le dispositif d'expérimentation sylvicole de Paracou en Guyane française, thèse, Paris 6, Paris, 1993.

[7] Cavelier J., Machado J.L., Valencia D., Montoya J., Laignelet A., Jurtado A., Varela A., Mejia C., Leaf demography and growth rates of Espeletia barclayaba Cuatrec. (Compositae), a caulescent rosette in a Colombian Paramo, Biotropica 24 (1) (1992) 52-63.

[8] Cebrian J., Duarte C.M., The dependance of herbivory on growth rate in natural plant communities, Funct. Ecol 8 (1994) 518-525.

[9] Coley P.D., Herbivory and defensive characteristics of tree species in a lowland tropical forest, Ecological Monographs 53 (2) (1983) 209-233.

[10] Coley P.D., Intraspecific variation in herbivory on two tropical tree species, Ecology 64 (3) (1983) 426-433.

[11] Collectifs, Atlas des départements franÿais d'Outre-Mer : 4. La Guyane, CNRS-Orstom, Paris, 1979.

[12] Devries P.J., Patterns of butterfly diversity and promising topics in natural history and ecology, in : Mooney H.A. (Ed.), Response of Plants to Multiple Stresses, Academic Press, New York, 1991, pp. 187-194.

[13] Dickson R.E., Isebrands J.G., Leaves as regulators of stress response, in : Mooney H.A. (Ed.), Response of Plants to Multiple Stresses, Academic Press Inc, London, 1991, pp. 4-34.

[14] Favrichon V., Classification des espèces arborées en groupes fonctionnels en vue de la réalisation d'un modèle de dynamique de peuplement en forêt guyanaise, Rev. Ecol. (Terre Vie) 49 (1994) 379-403.

[15] Fonseca C.R., Herbivory and the long-lived leaves of an Amazonian ant-tree, J. Ecol. 82 (1994) 833-842.

[16] Forget P.M., Regeneration Ecology of Eperua grandiflora (Caesalpiniaceae), a large-seeded tree in French Guiana, Biotropica 24 (1992) 146-156.

[17] Givnish T.J., Adaptation to sun and shade: a whole-plant perspective, Aust. J. Plant Physiol. 15 (1988) 63-92.

[18] Gleeson S.K., Tilman D., Plant allocation, growth rate and successional status, Funct. Ecol. 8 (1994) 543-550.

[19] Herms D.A., Mattson W.J., The dilemma of plants: to grow or defend, Q. Rev. Biol. 67 (3) (1992) 283-317.

[20] Janzen D.H., Sweep samples of tropical foliage insects: effects of seasons, vegetation types, elevation, time day, and insularity, Ecology 54 (1973) 687-708.

[21] Jones C.G., Coleman J.S., Plant stress and insect herbivory: toward an integrated perspective, in : Mooney H.A. (Ed.), Response of Plants to 
Multiple Stresses, Academic Press, New York, 1991, pp. 249-280.

[22] Koppel J.V. (van de), Patterns of herbivory along a productivity gradient: an empirical and theoretical investigation, Ecology 77 (3), (1996) 736-745.

[23] Kozlowski T.T., Kramer P.J., Pallardy S.G., The Physiological Ecology of Woody Plants, Academic Press, New York, 1991.

[24] Kursar T.A., Coley P.D., Delayed development of the photosynthetic apparatus in tropical rain forest species, Funct. Ecol. 6 (1992) 411-422.

[25] Lowman M.D., An assessment of techniques for measuring herbivory: Is rainforest defoliation more intense than we thought?, Biotropica 16 (4) (1984) 264-268.

[26] Lowman M.D., Herbivory as a canopy process in rain forest trees, in : Lowman M.D. (Ed.), Forest Canopies, Academic Press, New York, 1995, pp. 431-455.

[27] Marquis R.J., Phenological variation in the neotropical understory shrub Piper arieianum (Piperaceae): causes and consequences, Ecology 69 (1988) 1552-1565.

[28] Marquis R.J., Braker H.E., Plant-herbivore interactions: diversity, specificity, and impact, in : McDade L.A. (Ed.), La Selva, Ecology and Natural History of a Neotropical Rain Forest, University of Chicago Press, Chicago, 1991, pp. 261-281.

[29] Montpied P., La régénération naturelle en forêt tropicale humide : effets de traitements sylvicoles d'intensité variable, in : Engref (éd.), Actes du séminaire Silvolab, Recherches sur les écosystèmes forestiers de Guyane, Kourou, 1995, pp. 51-69.
[30] Newbery D.Mc.C., De Foresta H., Herbivory and defense in pioneer, gap and understory trees of tropical rain forest in French Guyana, Biotropica 17 (3) (1985) 238-244.

[31] Rhoades D.F., Évolution of plant chemical defense against herbivore, in : Rosenthal G.A., Janzen D.H. (Ed.), Herbivores : Their interactions with Secondary Plant Metabolites, Academic Press, New York, 1979, pp. 3-54.

[32] Ribeiro S.P., Pimenta H.R., Fernandes G.W., Herbivory by chewing and sucking insects on Tabebuia ochracea, Biotropica 26 (3) (1994) 302-307.

[33] Schmidt L., Bariteau M., Étude de la croissance et de la régénération naturelle. Dispositif de Paracou, Bois For. Trop. 220 (1990) 3-23.

[34] Shure D.J., Wilson L.A., Patch-size effects on plant phenolic in successional opening of the southern appalachians, Ecology 74 (1993) 55-67.

[35] Sterk F., Meer (van der) P., Bongers F., Herbivory in two rain forest canopies in French Guyana, Biotropica 24 (1) (1992) 97-99.

[36] Tuomi J., Toward integration of plant defence theories, Tree 7 (11) (1992) 365-367.

[37] Wolda H., Fluctuations in abundance of tropical insects, Am. Nat. 112 (1978) 1017-1045.

[38] Wolda H., Spatial and temporal variation in abundance in tropical animals, in: Sutton S.L. (Ed.) Tropical Rain Forest : Ecology and Management, Blackwell Scientific, Oxford, UK, 1983, pp. 93-105.

[39] Wright S.J., Schaik van C.P., Light and the phenology of tropical trees, Am. Nat. 143 (1) (1994) 192-199. 